Homology, Homotopy and Applications, vol.5(1), 2003, pp.49-52

\title{
CUBICAL ABELIAN GROUPS WITH CONNECTIONS ARE EQUIVALENT TO CHAIN COMPLEXES
}

\author{
RONALD BROWN AND PHILIP J. HIGGINS
}

(communicated by George Janelidze)

\begin{abstract}
The theorem of the title is deduced from the equivalence between crossed complexes and cubical $\omega$-groupoids with connections proved by the authors in 1981. In fact we prove the equivalence of five categories defined internally to an additive category with kernels.
\end{abstract}

\section{Introduction}

The theorem of the title is shown to be a consequence of the equivalence between crossed complexes and cubical $\omega$-groupoids with connections proved by us in [4]. We assume the definitions given in [4]. Thus this paper is a companion to others, for example $[\mathbf{8}]$, which show that a deficit of the traditional theory of cubical sets and cubical groups has been the lack of attention paid to the "connections", defined in [4]. Indeed the traditional degeneracies of cubical theory identify certain opposite faces of a cube, unlike the degeneracies of simplicial theory which identify adjacent faces. The connections allow for a fuller analogy with the methods available for simplicial theory by giving forms of 'degeneracies' which identify adjacent faces of cubes. They are used in [4] and [1] to give a definition of a 'commutative cube'.

Part of the interest of these results is that the family of categories equivalent to that of crossed complexes can be regarded as a foundation for a non-abelian approach to algebraic topology and the cohomology of groups. These results show that a form of abelianisation of these categories leads to well-known structures.

Note also the paper by Crans [5] in which Teisi reduce to a simpler form in the category of abelian groups.

\section{Crossed complexes internal to an additive category with ker- nels}

The basic elements of what we say next are well known, but are given for completeness.

Suppose we are given an action of a group $P$ on the right of a group $M$ such that the action $\phi: M \times P \rightarrow M$ is a morphism of groups. Then, as is well known,

Received December 11, 2002, revised February 12, 2003; published on February 24, 2003. 2000 Mathematics Subject Classification: 18D35, 18G35, 55U15.

Key words and phrases: chain complexes, cubical sets with connections, abelian group objects, crossed complexes, globular and cubical $\omega$-categories.

(c) 2003, Ronald Brown and Philip J. Higgins. Permission to copy for private use granted. 
the action is trivial. The proof is easy: let $m \in M, p \in P$. Then $m^{p}=\phi(m, p)=$ $\phi(m, 1) \phi(1, p)=m^{1} 1^{p}=m$. It follows that a crossed module internal to the category of groups is just a morphism of abelian groups.

We need to consider the more general case of crossed modules over groupoids. Recall from [6] that groupoids internal to the category of abelian groups are equivalent to morphisms of abelian groups. This is part of the following result.

Theorem. Let $\mathcal{A}$ be an additive category with kernels. The following categories, defined internally to $\mathcal{A}$, are equivalent.

$\mathbb{B}_{1}$ : The category of chain complexes.

$\mathbb{B}_{2}$ : The category of crossed complexes

$\mathbb{B}_{3}$ : The category of cubical sets with connections.

$\mathbb{B}_{4}$ : The category of cubical $\omega$-groupoids with connections.

$\mathbb{B}_{5}$ : The category of globular $\omega$-groupoids.

Proof. By working on the morphism sets, we can as usual assume that we are working in the category of abelian groups. Note that the theorem of the title follows from the equivalence $\mathbb{B}_{3} \simeq \mathbb{B}_{1}$.

$\mathbb{B}_{1} \simeq \mathbb{B}_{2}:$ By a chain complex we shall always mean a sequence of objects and morphisms $\delta: A_{n} \rightarrow A_{n-1}, n \geqslant 1$, such that $\delta \delta=0$. Let $C$ be a crossed complex internal to $\mathcal{A}$. The associated chain complex $\alpha C$ will be defined by

$$
\begin{aligned}
& (\alpha C)_{0}=C_{0}, \\
& (\alpha C)_{1}=\operatorname{Ker}\left(\delta_{0}: C_{1} \rightarrow C_{0}\right), \\
& (\alpha C)_{n}=C_{n}(0), n \geqslant 2 .
\end{aligned}
$$

The crossed complex $\beta A$ associated to a chain complex $A$ will be defined by

$$
\begin{aligned}
& (\beta A)_{0}=A_{0}, \\
& (\beta A)_{1}=A_{0} \times A_{1}, \\
& (\beta A)_{n}=A_{0} \times A_{n}, n \geqslant 2 .
\end{aligned}
$$

The groupoid structure on $\beta A$ in dimension 1 is defined as usual by $\delta_{0}=\operatorname{pr}_{1}, \delta_{1}=$ $\operatorname{pr}_{1}+\left(\partial \circ \operatorname{pr}_{2}\right)$, and with composition $(a, b)+(a+\partial b, c)=(a, b+c)$. The structure on $(\beta A)_{n}$ for $n \geqslant 2$ is that the only addition is $(a, b)+(a, c)=(a, b+c)$. The operation of $(\beta A)_{1}$ on $(\beta A)_{n}, n \geqslant 2$, is $(a, b)^{(a, c)}=(a+\partial c, b)$. This gives our first equivalence, between chain complexes and crossed complexes.

$\mathbb{B}_{2} \simeq \mathbb{B}_{3}:$ An equivalence between crossed complexes and cubical $\omega$-groupoids with connections internally to the category of sets is established in [4]. Although choices are involved in this, the end result is a natural equivalence. It follows that this can be applied internally to a category $\mathcal{A}$, simply by applying it to the morphism sets $\mathcal{A}(X, A)$ for all objects $X$ of $\mathcal{A}$. This yields our equivalence between crossed complexes and cubical $\omega$-groupoids with connections internal to $\mathcal{A}$.

$\mathbb{B}_{2} \simeq \mathbb{B}_{5}$ : This follows, in a similar way, from the equivalence between crossed complexes and globular $\omega$-groupoids proved in $[\mathbf{3}]$. (Reference $[\mathbf{2}]$ is relevant to the equivalence $\mathbb{B}_{1} \simeq \mathbb{B}_{5}$.) 
$\mathbb{B}_{3} \simeq \mathbb{B}_{4}$ : Let $K$ be a cubical abelian group with connections, in the sense of [4].

Lemma. If $G$ is an abelian group, and if $s, t: G \rightarrow G$ are endomorphism of $G$ such that $s t=s, t s=t$, then we can define a groupoid structure on $G$ with source and target maps $s, t$ by

$$
g \circ h=g-t g+h,
$$

for $g, h \in G$ with $t g=s h$, and this defines on $G$ the structure of groupoid internal to abelian groups.

This result comes from [6], and is also a special case of a non-abelian result on cat $^{1}$-groups $[\mathbf{7}]$, where the condition $[\operatorname{Ker} s$, Ker $t]=1$ is required, and is here trivially satisfied. This result can be applied to $K_{n}, n \geqslant 1$, and for each $i=1, \ldots, n$, with $s_{i}=\epsilon_{i} \partial_{i}^{0}, t_{i}=\epsilon_{i} \partial_{i}^{1}$, giving $n$ compositions and so a cubical complex with compositions and connections in the sense of $[\mathbf{1}, \mathbf{4}]$. The interchange law is easily verified, and there remains essentially only the transport law for the connections, which is again simple, showing that $K$ is now a cubical $\omega$-groupoid with connections. It is easy to see that the functor thus defined is adjoint to the forgetful functor $\mathbb{B}_{4} \rightarrow \mathbb{B}_{3}$.

\section{References}

[1] Al-Agl, F.A., Brown, R. And Steiner, R., 'Multiple categories: the equivalence between a globular and cubical approach', Advances in Mathematics 170 (2002) 71-118.

[2] Bourn, D., 'Another denormalization theorem for the abelian chain complexes', J. Pure Appl. Algebra 66 (1990) 229-249.

[3] Brown, R. And Higgins, P.J. , 'The equivalence of $\infty$-groupoids and crossed complexes', Cah. Top. Géom. Diff. 22 (1981) 371-386.

[4] Brown, R. and Higgins, P.J., 'On the algebra of cubes', J. Pure Appl. Algebra 21 (1981) 233-260.

[5] Crans, S.E., 'Teisi in Ab', Homology, Homotopy and Applications 3 (2001) $87-100$.

[6] Grothendieck, A., 'Catégories cofibrées additives et complexe cotangent relatif', Springer Lecture Notes in Math. 79 (1968) Springer-Verlag, Berlin, $167 \mathrm{pp}$.

[7] Loday, J.-L., 'Spaces with finitely many non-trivial homotopy groups', $J$. Pure Appl. Algebra 24 (1982) 179-202.

[8] Tonks, A.P., 'Cubical groups which are Kan', J. Pure Appl. Algebra 81 (1992) 83-87.

This article may be accessed via WWW at http://www.rmi.acnet.ge/hha/ or by anonymous ftp at

ftp://ftp.rmi.acnet.ge/pub/hha/volumes/2003/n1a2/v5n1a2.(dvi,ps,pdf) 
Ronald Brown r.brown@bangor.ac.uk

Mathematics Division

School of Informatics,

University of Wales, Bangor

Gwynedd LL57 1UT, U.K.

Philip J. Higgins p.j.higgins@durham.ac.uk

Department of Mathematical Sciences,

Science Laboratories,

South Rd.,

Durham, DH1 3LE, U.K. 